\title{
Expression analysis of VEGFA, FGF2, TGFß1, EGF and IGF1 in human nasal polyposis
}

\author{
APOSTOLOS ZARAVINOS $^{1}$, GIANNOULA SOUFLA ${ }^{1}$, JOHN BIZAKIS ${ }^{2}$ and DEMETRIOS A. SPANDIDOS ${ }^{1}$ \\ ${ }^{1}$ Laboratory of Virology, Medical School, University of Crete, Heraklion; ${ }^{2}$ Department of \\ Otolaryngology, University Hospital of Heraklion, Crete, Greece
}

Received November 12, 2007; Accepted December 18, 2007

\begin{abstract}
A better understanding of the expression profile of a group of angiogenic markers in nasal polyps (NPs) would contribute considerably to the investigation of the formation of NPs. The aim of this study was to evaluate the combined mRNA expression of vascular endothelial growth factor A (VEGFA), fibroblast growth factor 2 (FGF2), transforming growth factor $\beta 1$ (TGFß1), epidermal growth factor (EGF) and insulin-like growth factor 1 (IGF1) in NPs obtained from 21 patients undergoing nasal polypectomy. Nasal mucosae were obtained from the adjacent inferior turbinates (AIT) and middle turbinates (AMT) of the patients, as well as from 11 control subjects undergoing nasal corrective surgery. Analysis was performed using real-time RT-PCR. VEGFA, TGFß1 and IGF1 exhibited significant over-expression in the NPs compared to the control turbinates, EGF did not exhibit significant expression, and FGF2 presented constant overexpression in the NPs compared to both the adjacent and control turbinates. Since its mRNA levels were positively correlated with all the corresponding levels of the rest of the growth factors studied, TGFß1 seems to be a key cytokine in interactions between NP cells and the leading molecule of the epithelial differentiation and tissue remodelling present in the disease. Many correlations between the transcript levels of the other growth factors arose in the NP group as well, supporting a co-regulation of these genes in nasal polyposis. Our conclusions were that that VEGFA and TGFß1 participate significantly in the formation of NPs, whereas FGF2 and IGF1 are implicated in nasal polyposis to a lesser, but still significant, extent. EGF does not seem to be actively involved in the NP evolution process.
\end{abstract}

Correspondence to: Professor D.A. Spandidos, Laboratory of Virology, Medical School, University of Crete, Heraklion 71100, Crete, Greece

E-mail: spandidos@spandidos.gr

Key words: nasal polyps, gene expression, vascular endothelial growth factor A, fibroblast growth factor 2, transforming growth factor $\$ 1$, epidermal growth factor, insulin-like growth factor 1 , real-time PCR

\section{Introduction}

Nasal polyps (NPs) are a common chronic disease of the nasal or paranasal sinus mucosa characterized by proliferation of the epithelial layer, glandular hyperplasia, thickening of the basement membrane, edema, focal fibrosis and cellular infiltration of the stroma layer (1). Most often they originate from the middle meatus and the ethmoid sinus region of the nasal cavity (2). Nasal polyposis is almost always present in conjunction with chronic rhinosinusitis (CRS/NP) (3). The molecular alterations required for NP development have been under investigation only in recent years, and their molecular structure is still not completely understood.

Angiogenesis is an important event during the neoplastic process. This complex procedure, also known as neovascularization, is essential for tissue development, wound healing and reproduction (4), and is an indispensable requirement for tumor progression, invasiveness and metastasis (5). Tumor cells, as well as certain stromal cells such as macrophages, mast cells and fibroblasts, are known to secrete a large number of growth factors that activate neovascularization. However, the balance between angiogenic enhancers and endogenous inhibitors adjusts the angiogenic switch. Increasing evidence implicates angiogenesis not only in tumor growth but also in several chronic inflammatory disorders (6-8).

The induction of the angiogenic switch in several cancer types has been mainly associated with vascular endothelial growth factor A (VEGFA) and fibroblast growth factor 2 (FGF2) up-regulation, while transforming growth factor $B$ (TGFß) has been recognized as a growth inhibitor $(4,5)$. Although the mechanisms leading to the growth of NPs are unclear, the increased proliferation of epithelial cells, endothelial cells and fibroblasts may play an important role in the pathogenesis of NPs $(9,10)$. Polypeptide growth factors have been shown to stimulate cell growth in various in vitro and in vivo systems. It has been reported that some growth factors, including TGFß, platelet-derived growth factor, insulin-like growth factor 1 (IGF1) and basic fibroblastic growth factor (bFGF), may contribute to cellular proliferation in NPs (11-13), thus no real-time RT-PCR experiments have been conducted for the verification of their results. RT-PCR is an established technique for quantifying mRNA expression in biological samples. The benefits of this procedure over conventional methods include its sensitivity, large dynamic 
Table I. Clinicopathological characteristics of patients and control subjects.

\begin{tabular}{lcc}
\hline & $\begin{array}{c}\text { Nasal polyps and } \\
\text { adjacent turbinates }\end{array}$ & $\begin{array}{c}\text { Control } \\
\text { mucosae }\end{array}$ \\
\hline Subjects (no.) & $21^{\mathrm{a}}$ & 11 \\
Sex (M/F) & $15 / 6$ & $9 / 2$ \\
Age (years) & 51.2 & 33.4 \\
Range & $21-73$ & $12-68$ \\
Current or recent & $7 / 14$ & $3 / 8$ \\
ex-smokers (yes/no) & & $4 / 7$ \\
Alcohol (yes/no) & $6 / 15$ & $11 / 0$ \\
Aspirin & $5 / 16$ & \\
tolerance/intolerance & & \\
Stage of nasal polyp & & \\
Grade I & & \\
Grade II & & \\
Grade III & 10 & \\
\hline
\end{tabular}

range and potential for high, as well as accurate, quantification output.

In order to investigate the significance of angiogenic markers in NP formation, we evaluated the combined mRNA expression of VEGFA, FGF2, TGFß1, epidermal growth factor (EGF) and IGF1 in NPs in mucosae from the adjacent inferior turbinates (AIT) and middle turbinates (AMT) of the patients, as well as in control mucosae from the inferior and middle turbinates (CIT and CMT) of healthy subjects, using an RT-PCR assay with SYBR-Green I. We further examined whether the mRNA expression profile of these genes is correlated with the clinical stage of the disease and other clinicopathological features.

\section{Patients and methods}

Patients. Biopsies of the NPs and mucosa from the AIT and AMT were obtained from a total of 21 patients with CRS/NP (15 men and 6 women; average age, 51.2 years; range, 21-73 years). In patients 1-4, no middle turbinate was ceded. Following histopathological examination of the specimens, a grading system was used for each nasal cavity: I, small polyps located either in the middle meatus or superior to the middle turbinate; II, polyps extending between the upper and lower limits of the inferior turbinate; III, polyps reaching below the lower limit of the inferior turbinate (Table I). Moreover, patients were divided according to aspirin-tolerance $(n=5)$ and intolerance $(n=16)$ on the basis of history. All patients suffered from sneezing, rhinorrhea, headaches, nasal obstruction and hyposmia or anosmia, and had undergone nasal polypectomy at least once in the past. NP patients had not taken any drug (topical or systemic corticosteroids) within 4 weeks of surgery.

Eleven subjects with nasal septum deformity ( 9 men and 2 women; average age, 36.8 years; range, 12-68 years) who underwent nasal corrective surgery and had no history of nasal
Table II. Primer sequences used for quantitative RT-PCR.

\begin{tabular}{llc}
\hline Primer & \multicolumn{1}{c}{$\begin{array}{c}\text { Sequence } \\
\left(5^{\prime}-3^{\prime}\right)\end{array}$} & $\begin{array}{c}\text { Amplicon } \\
\text { pair size } \\
(\mathrm{bp})\end{array}$ \\
\hline EGF & $\begin{array}{l}\text { CTTGTCATGCTGCTCCTCCTG } \\
\text { TGCGACTCCTCACATCTCTGC }\end{array}$ & 118 \\
FGF2 & $\begin{array}{l}\text { CTGGCTATGAAGGAAGATGGA } \\
\text { TGCCCAGTTCGTTCAGTG }\end{array}$ & 149 \\
TGF31 & $\begin{array}{l}\text { AAGGACCTCGGCTGGAAGTGC } \\
\text { CCGGGTTATGCTGGTTGTA }\end{array}$ & 137 \\
VEGFA & $\begin{array}{l}\text { ATGACGAGGGCCTGGAGTGTG } \\
\text { CCTATGTGCTGGCCTTGGTGAG }\end{array}$ & 91 \\
& $\begin{array}{l}\text { CCTCCTCGCATCTCTTCTACCTGC } \\
\text { IGF1 }\end{array}$ & 166 \\
GAPDH & $\begin{array}{l}\text { GGAAGGTGAAGGTCGGAGTCA } \\
\text { GTCATTGATGGCAACAATATCCACT }\end{array}$ & 101 \\
\hline
\end{tabular}

or allergic symptoms of any nature served as our control group. During these operations, a biopsy from the mucosa of the CIT and CMT of each patient was acquired. No patient in this group had received corticosteroids for at least 1 month before surgery.

All study groups were submitted to serum determination of total IgE, specific IgE (RAST) and to hypersensitivity skin tests for a number of allergens; patients with allergies or asthma were excluded from the study. Clinical parameters such as age, sex, smoking and alcohol habits were available for all the patients and control subjects.

The Ethics Committee of the University of Crete approved the present study and all participants (patients and control subjects) were informed of its scope and gave their informed written consent.

RNA extraction and reverse transcription. Total-RNA was extracted from each specimen using a power homogenizer and TRIzol ${ }^{\circledR}$ reagent (Invitrogen, Carlsband, CA) according to the manufacturer's instructions. cDNA was synthesized using the StrataScript reverse transcriptase kit (Stratagene, La Jolla, CA) as previously described (14).

$R T$-PCR. Peptide growth factor mRNA expression was measured using RT-PCR with SYBR-Green I. Primers were designed to span introns. Glyceraldehyde-3-phosphate dehydrogenase (GAPDH) was used as the internal control in order to normalize VEGFA, TGFß1, FGF2, EGF and IGF1 expression levels. Specifically, $1 \mu 1$ cDNA from NP, AMT, AIT, CMT or CIT samples was amplified in a PCR reaction containing 2X Brilliant SYBR-Green I QPCR Master Mix, $300 \mathrm{nM}$ of each primer and $30 \mu \mathrm{M}$ ROX passive reference dye, in a final volume of $20 \mu \mathrm{l}$. After an initial denaturation at $95^{\circ} \mathrm{C}$ for $10 \mathrm{~min}$, the samples were subjected to 40 cycles of amplification comprised of denaturation at $95^{\circ} \mathrm{C}$ for $30 \mathrm{sec}$, annealing at $60^{\circ} \mathrm{C}$ for $30 \mathrm{sec}$ and elongation at $72^{\circ} \mathrm{C}$ for $30 \mathrm{sec}$, followed by a melt curve analysis in which the temperature was increased from $55^{\circ} \mathrm{C}$ to $95^{\circ} \mathrm{C}$ at a linear rate of $0.2^{\circ} \mathrm{C} / \mathrm{sec}$. Data collection was performed during both annealing and 
A.

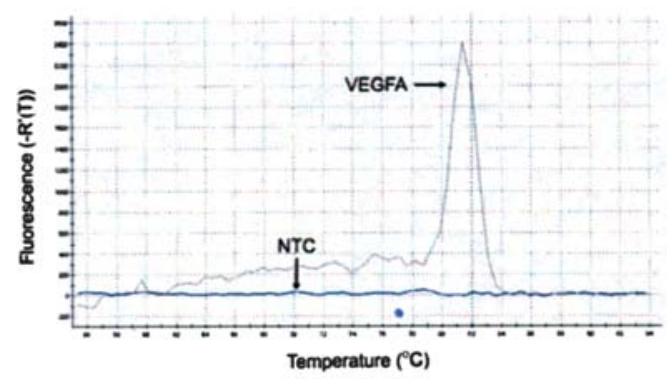

C.

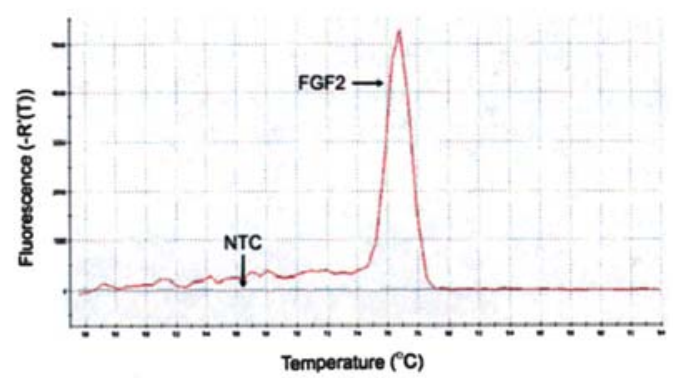

E.

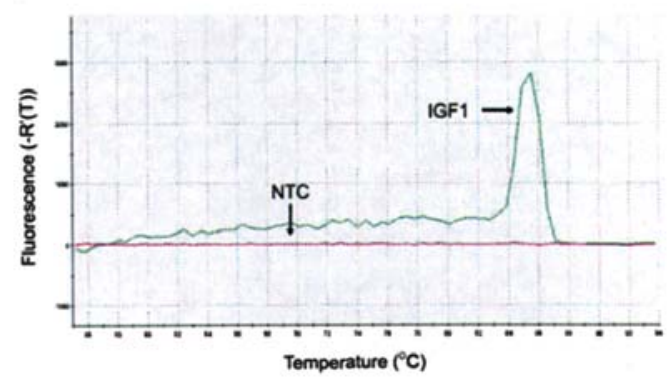

B.

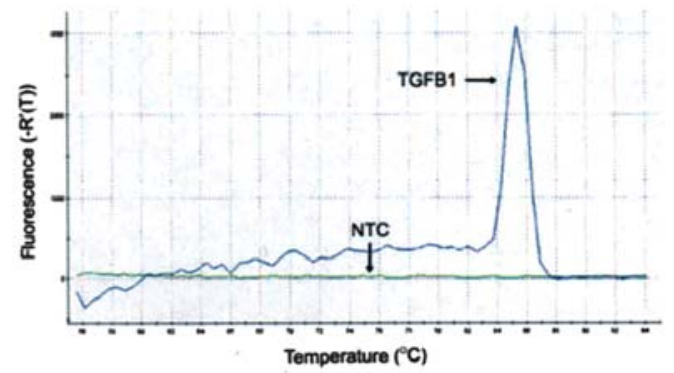

D.

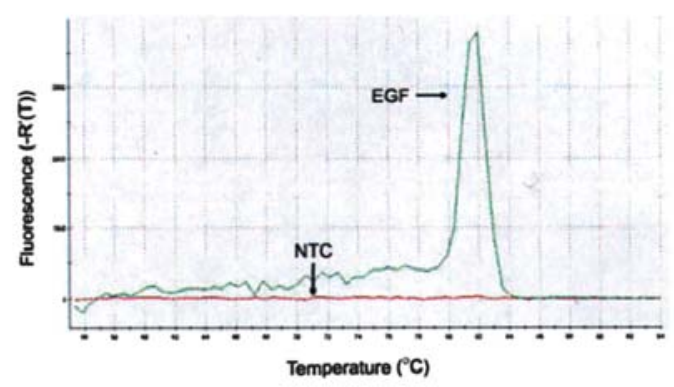

F.

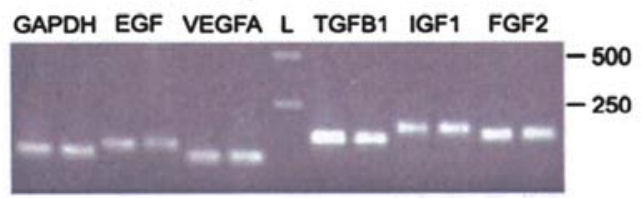

Figure 1. Dissociation curves of (A) VEGFA, (B) TGFß1, (C) FGF2, (D) EGF, (E) IGF1 and (F) gel analysis of all the genes. Single peaks and bands of representative samples prove the specificity of the amplification products, as well as the absence of any by-product or primer-dimer formation. The $\mathrm{T}_{\mathrm{m}}$ of each amplification product is indicated with the dissociation peak. NTC, non-template control.

extension with 2 measurements at each step, and at all times during the melt curve analysis. All PCR experiments were conducted on the Mx3000P RT-PCR thermal cycler using software version 2.0 (Stratagene). In order to verify the results of the melt curve analysis, PCR products were analyzed by electrophoresis in $2 \%$ agarose gel, stained with ethidium bromide and photographed on a UV light trans-illuminator. PCR product lengths for all the growth factors analyzed as well as for GAPDH are depicted in Table II. In each PCR reaction, 2 non-template controls were included.

All reactions were run in triplicate and peptide growth factor transcript levels were calculated and normalized to each specimen's housekeeping gene mRNA (GAPDH), as well as to the appropriate calibrators, using the $\Delta \Delta \mathrm{Ct}$ method for relative quantification. Specifically, after amplification, standard curves were constructed from samples used in a series of consecutive dilutions for both the gene of interest (GF) and the internal control (GAPDH). Growth factor and GAPDH amplification efficiencies were the same, reaching $100 \%$. $\mathrm{NP}$, adjacent and control turbinate data were first normalized against variation in sample quality and quantity. Normalized values to GAPDH, $\Delta \mathrm{Cts}$, were initially calculated using the following equation: $\Delta \mathrm{Ct}_{\text {sample }}=\mathrm{Ct}_{\mathrm{GF}}-\mathrm{Ct}_{\mathrm{GAPDH}}$. The $\Delta \Delta \mathrm{Ct}$ was then determined using the formula $\Delta \Delta \mathrm{Ct}=\Delta \mathrm{Ct}_{\mathrm{NP}}-\Delta \mathrm{Ct}_{\text {turbinate }}$ and the expression of the normalized (to GAPDH) GF genes in NP compared to the corresponding turbinate used as a calibrator $=2^{-\Delta \Delta \mathrm{Ct}}$. A 2-fold increased $(\geq 2)$ or decreased $(\leq 0.5)$ value compared to the calibrator was considered GF mRNA over-expression or down-regulation, respectively.

Statistical analysis. Peptide growth factor mRNA levels were first evaluated by the non-parametric Friedman's test for several related samples, and by the Wilcoxon test for the 2-related samples test. The non-parametric Spearman rank correlation was used to examine their relation pair-wise and their association with continuous variables (age, stage of NP). All statistical analyses were performed with SPSS 11.5 (SPSS, Chicago, IL). Statistical significance was set at the $95 \%$ level $(\mathrm{P}<0.05)$.

\section{Results}

This study was conducted in order to determine mRNA transcription levels of VEGFA, TGFß1, FGF2, EGF and IGF1 peptide growth factors using the quantitative RT-PCR method (Fig. 1). 
A.

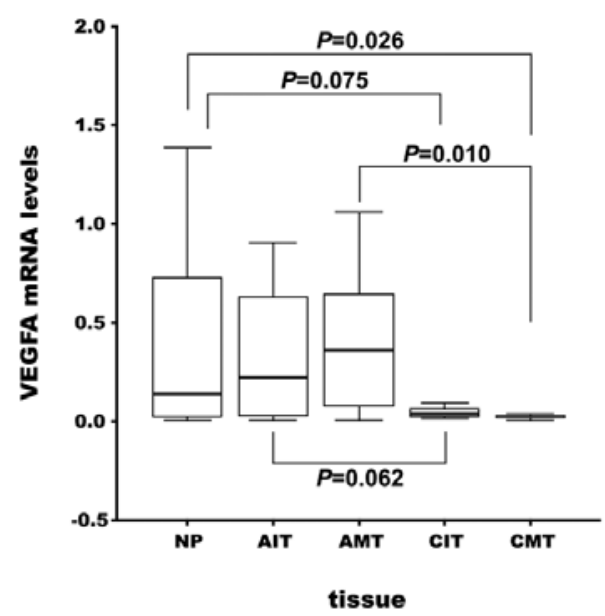

C.

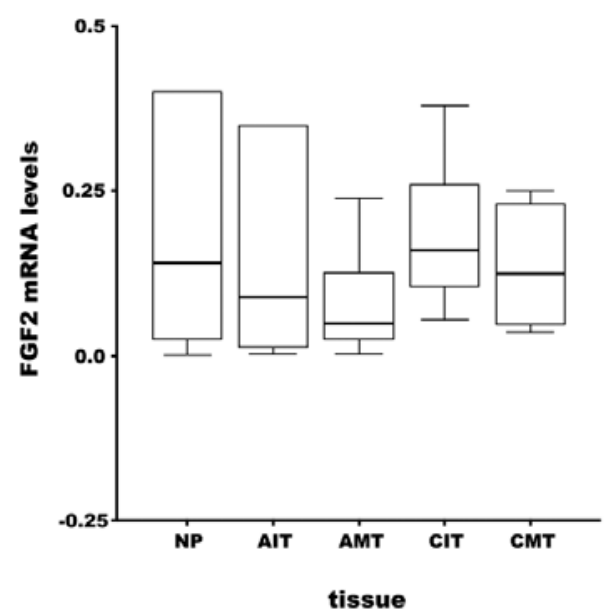

E.

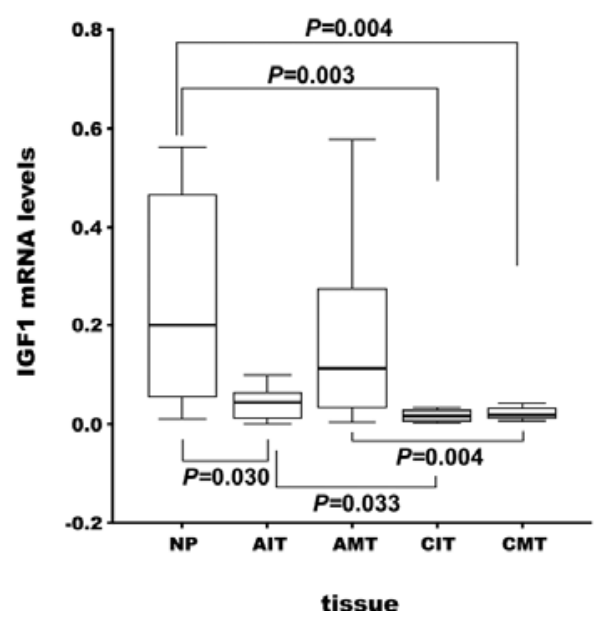

We initially calculated the normalized values of all target genes to GAPDH for all tissues, NPs, AITs, AMTs, CITs and CMTs, and compared the expression levels of GFs among the different tissue groups. Following this analysis, we used two approaches to estimate GF over- or under-expression in NPs. We calculated the expression of the normalized (to GAPDH) GF genes in NP compared to a) the corresponding AIT and AMT, used as the first calibrator and b) the average
B.

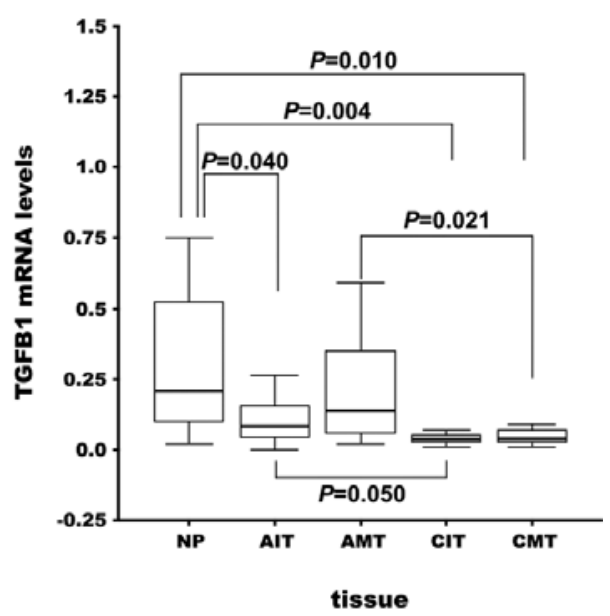

D.

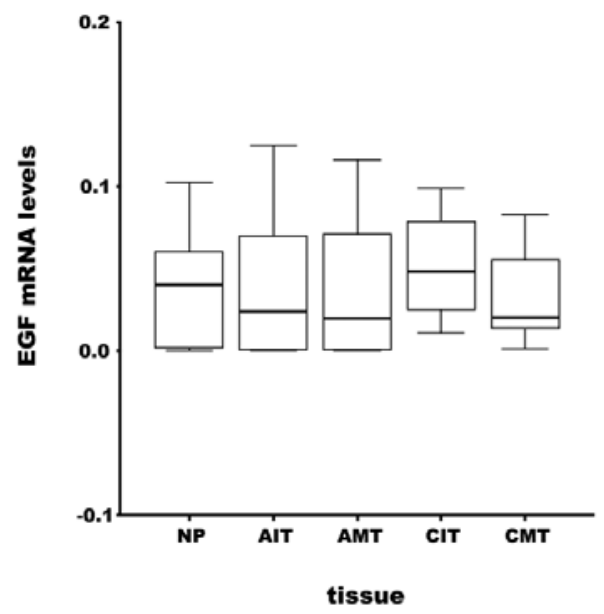

Figure 2. mRNA levels of (A) VEGFA, (B) TGFß1, (C) FGF2, (D) EGF and (E) IGF1 in the nasal polyps (NPs), adjacent inferior/middle turbinates (AIT/AMTs) and the control inferior/middle turbinates (CIT/CMTs). Relative values of growth factor expression vs. GAPDH exhibited significant differences with respect to tissue type $(\mathrm{P}<0.070$ for VEGFA, $\mathrm{P}=0.005$ for TGFß1 and $\mathrm{P}<0.001$ for IGF1, respectively; Friedman's test). Significant differences, assessed by the Wilcoxon test for 2-related samples test, are shown. Boxplots show the 25th, 50th (median) and 75th percentile values. Whiskers show the minimum and maximum values.

normalized values of all CIT and CMT samples, used as the second calibrator.

Expression levels. Regarding the expression levels of the peptide growth factors in the tissues studied, VEGFA and TGFß1 were expressed in all the tissues. FGF2 was expressed in $86 \%$ of NPs, $86 \%$ of AITs and $82 \%$ of AMTs. All CIT and $90 \%$ CMT presented FGF2 expression. EGF exhibited 


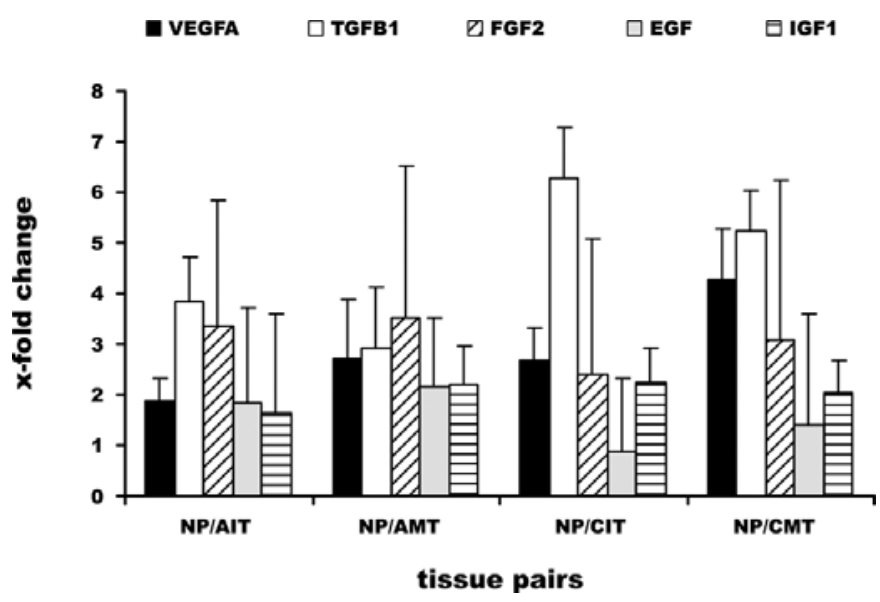

Figure 3. X-fold change in expression levels of VEGFA, TGFß1, FGF2, EGF and IGF1 between the nasal polyp and adjacent (NP/AIT, NP/AMT) or control (NP/CIT, NP/CMT) turbinates. VEGFA, TGFß1, EGF and IGF1 mRNA expression was significantly higher in NPs relative to control turbinates and in NPs relative to adjacent turbinates. Values shown are the mean $\pm \mathrm{SD}$.

expression in $66 \%$ of NPs, $74 \%$ of AITs, $75 \%$ of AMTs and in all control turbinates. Finally, IGF1 showed expression in 95\% of NPs, $90 \%$ of AITs and in all AMTs and control turbinates.

According to the first part of our analysis, VEGFA, TGFß1 and IGF1 normalized mRNA expression levels presented significant differences among the tissues studied (NP, AIT, AMT, CIT and CMT $)(\mathrm{P}<0.070, \mathrm{P}=0.005$ and $\mathrm{P}<0.001$, respectively; Friedman's test) (Fig. 2), whereas EGF and FGF2 did not. The mean expression values for VEGFA, TGFß1 and IGF1 were higher in the polyps compared to the CMTs $(\mathrm{P}=0.026, \mathrm{P}=0.010$ and $\mathrm{P}=0.004$, respectively; Wilcoxon test $)$, and compared to the CITs in the case of TGFß1 and IGF1 only $(\mathrm{P}=0.004$ and $\mathrm{P}=0.003$, respectively; Wilcoxon test). Of note, TGFß1 mRNA levels were higher in the polyps compared to just the AITs ( $\mathrm{P}=0.040$, Wilcoxon test). Moreover, the AITs presented higher VEGFA, TGFß1 and IGF1 mRNA levels than the corresponding CITs $(\mathrm{P}=0.062$, marginal, $\mathrm{P}=0.050$ and $\mathrm{P}=0.033$, respectively), whereas the AMTs compared to the CMTs presented significantly elevated levels of VEGFA, TGFß1 and IGF1 $(\mathrm{P}=0.010, \mathrm{P}=0.021$ and $\mathrm{P}=0.004$, respectively). FGF2 and EGF presented similar expression levels among all the tissues.

In the second part of our analysis, we estimated GFs overand under-expression in NPs by using different calibrators, the adjacent and control (inferior and middle) turbinates. Fig. 3 depicts the mean fold change in the mRNA expression levels of all GFs in NPs according to each calibrator. VEGFA and TGFß1 mRNA fold change was higher in NPs relative to the control turbinates than it was in NPs relative to the adjacent turbinates (for TGFß1: NP/AIT-NP/CIT, $\mathrm{P}=0.043$ and NP/AMT-NP/CMT, P=0.008, Mann-Whitney). FGF2, EGF and IGF1 fold change was similar among the tissue pairs NP/AIT, NP/AMT, NP/CIT and NP/CMT. Thus, the fold change in mRNA levels of VEGFA and TGFß1 displays a different expression pattern depending on whether the calibrator used is the adjacent (NP/AIT/AMT) or control turbinate (NP/CIT/ CMT).
Growth factor expression levels compared to clinicopathological characteristics. No statistically significant correlation was established between GF mRNA levels, sex, age, smoking or aspirin tolerance/intolerance. NP occurrence was more frequent in men than in women $\left(\mathrm{P}=0.003 ; \chi^{2}\right)$ and patients over 40 years old were more prone to the disease $\left(\mathrm{P}=0.022, \chi^{2}\right)$.

VEGFA mRNA levels presented a significant difference in terms of the stage of the NP $(\mathrm{P}=0.05)$. VEGFA mRNA levels were higher in grade I NPs compared to grade II $(\mathrm{P}=0.015)$ and III polyps. Regarding TGFß1, grade I NPs presented marginally elevated expression levels compared to grade II $(\mathrm{P}=0.066)$, and significantly elevated levels compared to grade III, polyps $(\mathrm{P}=0.043)$. However, levels in grade II polyps were not higher than they were in grade III ones, suggesting that the cause is random variation and not stage effect. FGF2, EGF and IGF1 mRNA levels rose proportionally to the stage of the NP, but the difference was not statistically significant.

Pair-wise mRNA expression analysis. Significant Spearman correlations between the co-expression patterns of all the growth factors were assessed in the NP tissues. Specifically, TGFß1 expression levels were significantly correlated with the corresponding levels of VEGFA $(\mathrm{P}=0.045)$, FGF2 $(\mathrm{P}=0.021)$, EGF $(\mathrm{P}=0.024)$ and IGF1 $(\mathrm{P}=0.027)$. Moreover, in the NP group, FGF2 was well correlated with EGF $(\mathrm{P}=0.005)$ and IGF1 $(\mathrm{P}=0.018)$ transcript levels. Only a few correlations between the co-expression patterns of the growth factors were detected in the AMTs (VEGFA with EGF, $\mathrm{P}=0.042$ ) and the CITs (VEGFA with TGFß1, $\mathrm{P}=0.031$; EGF with FGF2, $\mathrm{P}=0.047$ ), and no significant correlations were detected in the AITs or CMTs. These findings imply that the genes are coregulated in the context of the disease.

\section{Discussion}

The goal of this study was to identify the expression profile of specific growth factor genes using RT-PCR in order to contribute considerably to the understanding of the formation of human NPs. This is the first evaluation of the gene expression profile of growth factors via RT-PCR in NPs compared to mucosa from the adjacent turbinates of the patients and to turbinates from symptom-free subjects. Lee et al (15) recently obtained a transcriptome of normal human nasal mucosa by SAGE, and 47 genes presented at least 2-fold higher expression in the NPs compared to the normal nasal mucosae, including growth factors. Our results are in accordance with their report regarding the expression of growth factor transcript levels in NPs, providing, for the first time, expression data on a specific group of growth factor genes.

Expression levels. All growth factors exhibited expression levels in the majority of NPs and turbinate mucosae except for EGF. VEGFA, TGFß1, FGF2 and IGF1 exhibited significant over-expression in the NPs relative to the turbinate mucosae, whereas EGF did not exhibit significant expression in the NPs.

The fold change in the expression of VEGFA and TGFß1 was higher in the NP-control turbinate tissue pairs than in the NP-adjacent turbinate tissue pairs. This implies that the mRNA levels of these factors are significantly elevated in the adjacent 
turbinate mucosae as well. In all previously published studies, the expression levels of various growth factors in NP tissues were compared to the corresponding levels in control mucosae (from patients undergoing nasal septum deformity). To our knowledge, this is the first report stating that the remodelling process also affects the adjacent mucosa in addition to the polyp tissue.

Growth factors have been shown to be expressed in NPs $(11,17,18)$ mainly by protein-based assays. The intense expression of VEGF in polyp cells should not be surprising since inflammatory cells, especially macrophages, intensely express VEGF mRNA in various inflammatory states (18). Coste et al (17) reported that NP epithelial cells are also able to express VEGF mRNA in vitro, and secrete the VEGF protein upon up-regulation by TGFß1. The VEGFA mRNA over-expression that we found in NPs relative to CITs and CMTs could therefore be attributed to both the inflammatory and epithelial cells of the polyps, as a response to high levels of TGFß1. TGFß1 mRNA levels were also significantly elevated in the polyps. This hypothesis is reinforced by our finding that, in the NPs, TGFB1 transcript levels were correlated to those of VEGFA. The elevated VEGFA mRNA expression that we observed in grade I, as opposed to grade II or grade III polyps, led us to surmise that VEGFA mRNA levels play a more active role at the beginning of the disease than its later stages, and are responsible for the induction of angiogenesis and increased microvascular permeability. However, the small number of grade I polyps and the observation that levels in grade II were not higher than in grade III does not allow us to draw final conclusions regarding the effect of NP VEGFA stage.

Previous studies $(11,19)$ have demonstrated elevated TGFß protein levels in NPs, suggesting that TGFß synthesized by eosinophils contributes to the structural abnormalities, such as stromal fibrosis, angiogenesis, epithelial and glandular hyperplasia and basement membrane thickening, which characterize this disease. In our study, and supporting the conclusions of protein-based reports $(11,19,20)$, TGFß1 presented mRNA over-expression in NPs compared to the turbinate mucosae. TGFß1 mRNA levels were also higher in grade I polyps than those of the more advanced stages. Nonetheless, the case is similar to that of VEGFA and seems to be caused by random variation and not by stage effect. By virtue of its ability to up-regulate VEGFA and to correlate positively with all the growth factors studied in the polyps, as opposed to the adjacent and control turbinates, TGFß1 seems to be a key cytokine in the interactions between NP cells, leading to the epithelial differentiation and tissue remodelling that is present in the disease.

Kim et al (21) reported, based on RT-PCR, that FGF2 is expressed in NPs more frequently than in inferior turbinates at both the protein and mRNA levels. In our study, FGF2 was present in approximately $86 \%$ of polyps, in contrast with Kim et al who reported a $29 \%$ mRNA expression of FGF2 in NPs. Apparently, this difference is attributed to the specificity of RT-PCR compared to semi-quantitative RT-PCR. In our study, FGF2 was over-expressed in 36.8 and $25 \%$ of NPs compared to AIT and AMT, respectively, and in only $19 \%$ of the polyps compared to both CIT and CMT. However, there was also a significant percentage of FGF2 under-expression in the NPs compared to the control turbinates (AIT, 33.3\% and AMT, 28.5\%). Our findings, that FGF2 mRNA expression levels are significantly correlated with the corresponding TGFß1 levels in polyps, are in accordance with those of Gajdusek et al (22), who suggested that FGF2 and TGFß1 comprise synergistic mediators of angiogenesis in vitro. Furthermore, the co-expression of FGF2 mRNA in polyps with EGF and IGF1, demonstrated in our study, reinforces the hypothesis that FGF2 acts as a paracrine mediator that facilitates NP growth by stimulating epithelial and endothelial proliferation. Grade III polyps presented slightly elevated FGF2, EGF and IGF1 mRNA expression levels (though these were not statistically significant) compared to levels at earlier stages of polyposis, leading us to assume that FGF2, EGF and IGF1 are not as critical to the induction of the disease as VEGFA and TGFß1 are, but provide assistance so that the modifications of epithelial differentiation and tissue remodeling can become more crucial in grades II and III.

This is the first attempt to evaluate the expression pattern of EGF in NPs via RT-PCR, and it seems that it is not significantly associated with the pathogenesis of the disease as it presents similar levels of expression in the pathologic (polyp) and non-pathologic (turbinate) tissues. On the other hand, it has been proposed that its receptor (EGFR) is correlated with the disease to a greater extent, since it has been shown to be increased in the epithelium, gland cells and inflammatory cells of NPs (23).

High concentrations of the tropic peptide IGF1 have been demonstrated by immunohistochemistry in NPs (24), and it has been proposed that nasal mucosal inflammatory reactions induce the local formation and accumulation of IGF1. This could eventually result in very high IGF1 concentrations in the paranasal sinuses due to their tendency to be enclosed, i.e. to produce reduced drainage. According to our evaluation, elevated IGF1 mRNA expression levels ( 2-fold) are also observed in NPs compared to adjacent and control turbinates.

In conclusion, tissue remodeling seems to be a prominent feature of NP formation and involves angiogenesis-related growth factors. Our data imply that the remodelling process affects not just the polyp tissue, but also the adjacent mucosa. Significant correlations between the co-expression patterns of all the growth factors were assessed in the NP tissues, implying a co-regulation of these genes in the context of the disease. Of all the genes studied, TGFß1 seems to be a key cytokine for the formation of NPs as it is correlated to all the other growth factors studied. The intense expression of VEGFA in polyp cells is justified since inflammatory cells, especially macrophages, intensely express VEGF mRNA in various inflammatory states. TGFß1 synthesized by eosinophils contributes to the structural abnormalities, such as stromal fibrosis, angiogenesis, epithelial and glandular hyperplasia and basement membrane thickening, which characterize nasal polyposis. FGF2, acting as a paracrine mediator, facilitates NP growth by stimulating epithelial and endothelial proliferation. EGF does not appear to be strongly associated with the pathogenesis of the disease, in contrast to its receptor. Finally, nasal mucosal inflammatory reactions seem to induce the local formation and accumulation of IGF1. Our findings provide new perspectives on the molecular mechanisms involved in the pathogenesis of NPs. However, further investigation 
needs to be conducted if new molecular targets of therapeutic strategies are to be identified.

\section{References}

1. Settipane GA, Lund VJ, Bernstein JM and Tos M (eds): Nasal Polyps: Epidemiology, Pathogenesis and Treatment. OceanSide Publications Inc., Providence, RI, 1997.

2. Larsen PL and Tos M: Origin of nasal polyps. Laryngoscope 101: 305-312, 1991.

3. Fokkens W, Lund V, Bachert C, et al: EAACI position paper on rhinosinusitis and nasal polyps executive summary. Allergy 60: 583-601, 2005.

4. Folkman J: What is the evidence that tumors are angiogenesis dependent? J Natl Cancer Inst 82: 4-6, 1990.

5. Carmeliet $\mathrm{P}$ and Jain RK: Angiogenesis in cancer and other diseases. Nature 407: 249-257, 2000.

6. Hoshino M, Nakamura Y and Hamid QA: Gene expression of vascular endothelial growth factor and its receptors and angiogenesis in bronchial asthma. J Allergy Clin Immunol 107: 1034-1038, 2001.

7. Chetta A, Zanini A, Foresi A, et al: Vascular component of airway remodeling in asthma is reduced by high dose of fluticasone. Am J Respir Crit Care Med 167: 751-757, 2003.

8. Wittekindt C, Hess A, Bloch W, Sultanie S and Michel O: Immunohistochemical expression of VEGF and VEGF receptors in nasal polyps as compared to normal turbinate mucosa. Eur Arch Otorhinolaryngol 259: 294-298, 2002.

9. Nakagawa T, Yamane H, Nakai Y, Shigeta T, Takashima T and Takeda Z: Comparative assessment of cell proliferation and accumulation of extracellular matrix in nasal polyps. Acta Otolaryngol 538 (Suppl): 205-208, 1998.

10. Coste A, Rateau JG, Bernaudin JF, Peynegre R and Escudier E: Nasal polyposis pathogenesis: a flow cytometric and immunohistochemical study of epithelial cell proliferation. Acta Otolaryngol 116: 755-761, 1996.

11. Elovic A, Wong DT, Weller PF, Matossian K and Galli SJ: Expression of transforming growth factors-alpha and beta 1 messenger RNA and product by eosinophils in nasal polyps. J Allergy Clin Immunol 93: 864-869, 1994.

12. Coste A, Wang QP, Roudot-Thoraval F, et al: Epithelial cell proliferation in nasal polyps could be up-regulated by plateletderived growth factor. Laryngoscope 106: 578-583, 1996.
13. Petruson B, Hansson HA and Petruson K: Insulin-like growth factor I immunoreactivity in nasal polyp. Arch Otolaryngol Head Neck Surg 114: 1272-1275, 1988.

14. Zaravinos A, Bizakis J, Soufla G, Sourvinos G and Spandidos DA: Mutations and differential expression of the ras family genes in human nasal polyposis. Int J Oncol 31: 1051-1059, 2007.

15. Lee JY, Lee SH, Lee HM, et al: Analysis of gene expression profiles of normal human nasal mucosa and nasal polyp tissues by SAGE. J Allergy Clin Immunol 118: 134-142, 2006.

16. Szefler SJ: Airway remodelling: therapeutic target or not? Am J Respir Crit Care Med 171: 672-673, 2005.

17. Coste A, Brugel L, Maitre B, Boussat S, Papon JF, Wingerstmann L, Peynegre R and Escudier E: Inflammatory cells as well as epithelial cells in nasal polyps express vascular endothelial growth factor. Eur Respir J 15: 367-372, 2000.

18. Dvorak HF, Detmar M, Claffey KP, Nagy JA, van de Water L and Senger DR: Vascular permeability factor/vascular endothelial growth factor: an important mediator of angiogenesis in malignancy and inflammation. Int Arch Allergy Immunol 107: 233-235, 1995.

19. Ohno I, Lea RG, Flanders KC, et al: Eosinophils in chronically inflamed human upper airway tissues express transforming growth factor $\beta 1$ gene (TGF-ß). J Clin Invest 89: 1662-1668, 1992.

20. Serpero L, Petecchia L, Sabatini F, Giuliani M, Silvestri M, Di Blasi P, Rossi GA: The effect of transforming growth factor (TGF)-beta1 and (TGF)-beta2 on nasal polyp fibroblast activities involved upper airway remodeling: modulation by fluticasone propionate. Immunol Lett 105: 61-67, 2006.

21. Kim HJ, Jung HH and Lee SH: Expression of acidic fibroblast growth factor and basic fibroblast growth factor in nasal polyps. Acta Otolaryngol 126: 600-605, 2006.

22. Gajdusek CM, Luo Z and Mayberg MR: Basic fibroblast growth factor and transforming growth factor beta-1: synergistic mediators of angiogenesis in vitro. J Cell Physiol 157: 133-144, 1993.

23. Burgel PR, Escudier E, Coste A, et al: Relation of epidermal growth factor receptor expression to goblet cell hyperplasia in nasal polyps. J Allergy Clin Immunol 106: 705-712, 2000. 